\title{
Atomically Resolved Chemical Reactivity of Small Fe Clusters
}

\author{
Julian Berwanger $\odot,{ }^{1,}{ }^{*}$ Svitlana Polesya, ${ }^{2}$ Sergiy Mankovsky, ${ }^{2}$ Hubert Ebert, ${ }^{2}$ and Franz J. Giessibl ${ }^{1}$ \\ ${ }^{1}$ Institute of Experimental and Applied Physics, University of Regensburg, 93040 Regensburg, Germany \\ ${ }^{2}$ Department of Chemistry, Ludwig-Maximilians-University Munich, 81377 Munich, Germany
}

(Received 24 September 2019; accepted 17 January 2020; published 4 March 2020)

\begin{abstract}
Small metal clusters have been investigated for decades due to their beneficial catalytic activity. It was found that edges are most reactive and the number of catalytic events increases with the cluster's size. However, a direct measurement of chemical reactivity of individual atoms within the clusters has not been reported yet. We combine the high-resolution capability of CO-terminated tips in scanning probe microscopy with their ability to probe chemical binding forces on single Fe atoms to study the chemical reactivity of atom-by-atom assembled Fe clusters from 1 to 15 atoms on the atomic scale. We find that the chemical reactivity of individual atoms within flat Fe clusters does not depend on the cluster size but on the coordination number of the investigated atom. Furthermore, we explain the atomic contrast of the investigated Fe clusters by relating the force spectra of individual atoms with atomic force microscopy images of the clusters.
\end{abstract}

DOI: 10.1103/PhysRevLett.124.096001

Since the concept of catalytic, highly reactive active sites on surfaces was introduced [1], many studies have been conducted to precisely locate them on various catalytic materials. Observations of the adsorption positions of reactants and reaction products have shown that step edges are catalytically most active for, e.g., the $\mathrm{Ru}(0001)$ surface [2], Pt clusters on graphene/Rh(111) [3], and the $\mathrm{Pt}(111)$ surface [4]. Furthermore, it was shown that catalytically inactive surfaces can be activated by integrating single $\mathrm{Pd}$ atoms in a $\mathrm{Cu}(111)$ surface [5] and that the catalytic activity of $\mathrm{Au}$ clusters on a $\mathrm{MgO}$ substrate can be enhanced by locally charging the substrate [6].

$\mathrm{Fe}$ is an essential catalytic material, which has the advantage that it is relatively nontoxic, affordable, abundantly available, and sustainable in contrast to many transition metals $[7,8]$. Furthermore, Fe is frequently used in nature as a biocatalyst and, hence, it is important in, e.g., organic synthesis $[8,9]$. So far, the chemical reactivity of Fe clusters was only studied as a function of the cluster size [10] and mainly in the gas phase [11-13]. In essence, it was found that the rate of chemical reactions increases with the number of atoms the Fe clusters consist of $[10,12,13]$. However, these collective studies did not reveal the chemical reactivity on the atomic scale and, hence, a localization of the active sites within a cluster is missing.

Terminating the metal tip in noncontact atomic force microscopy (NCAFM) [14] with a single carbon monoxide (CO) molecule $[15,16]$ allows resolving small Fe clusters [17], next to various other sample systems [16,18-22], on the atomic scale. Moreover, it was recently shown that a COterminated tip (CO tip) can undergo both a physisorbed and a chemisorbed binding state at two specific distances when approaching the center of a single $\mathrm{Fe}$ adatom on the $\mathrm{Cu}(111)$ surface [23]. It is important to note that $\mathrm{CO}$ tips that image $\mathrm{Fe}$ adatoms form medium strength chemical bonds, in stark contrast to CO tips imaging organic molecules $[16,24,25]$ or Si $[17,23]$ where Pauli repulsion prevails.

In this Letter, we are combining the ability of NCAFM with CO tips to assemble small Fe clusters atom by atom in a controlled fashion [26] with its unprecedented atomic resolution capability to measure the vertical short-range force $\left[F_{z, \mathrm{SR}}\left(z, c^{*}\right)\right]$ on manually created $\mathrm{Fe}$ clusters of different sizes (3-15 atoms). $F_{z, \mathrm{SR}}\left(z, c^{*}\right)$ is a function of distance $z$ and $c^{*}$, the reduced coordination number that refers to the neighboring cluster atoms which can be expressed by using the atom's coordination number $c$ via $c^{*}=c-3$ (see Sec. SP1 [27]). Upon approaching the tip, we observe the formation of a weak physical bond at larger distances, followed by a chemical bond between the $\mathrm{CO}$ tip and the cluster's individual atoms at close distances when the tip is positioned above the center of a cluster atom (similar to the situation for single Fe adatoms [23]). Here, we find that the strength of the chemical bond decreases with the atom's coordination number. Hence, it is strongest for a single $\mathrm{Fe}$ adatom. This finding agrees with the identification of step edges as the active sites for various materials [2-4]. We determine the local chemical reactivity within a Fe cluster by an atom-by-atom measurement of chemical bonding strength. Finally, we relate the $F_{z, \mathrm{SR}}\left(z, c^{*}\right)$ spectra to NCAFM images acquired in constant-current and constant-height mode to explain the contrast formation of $\mathrm{Fe}$ clusters using $\mathrm{CO}$ tips. The interpretation of our results is supported by accompanying density-functional theory (DFT).

Figure 1 displays the tip dependence of chemical reactivity by comparing three short-range force vs distance 


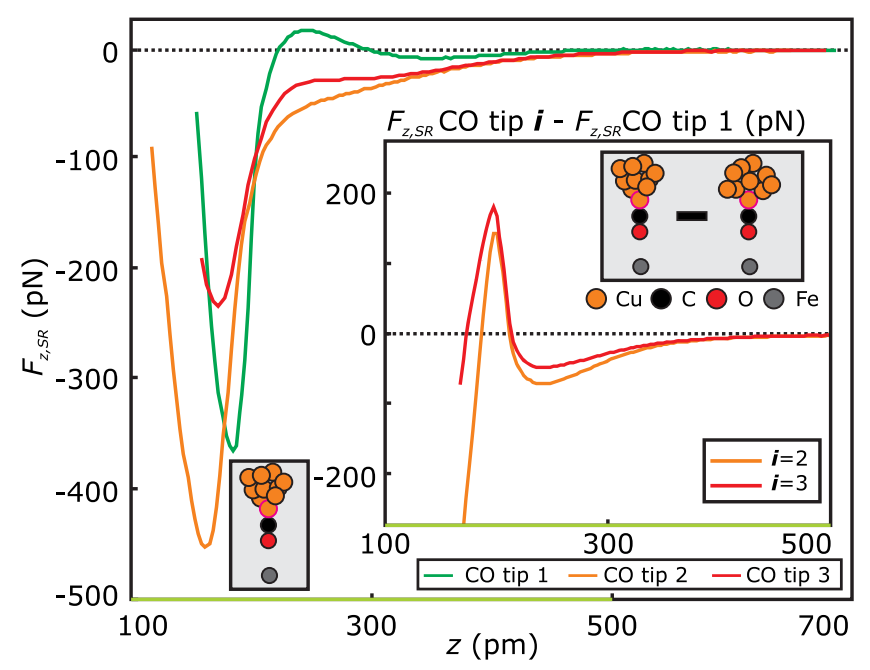

FIG. 1. Short-range force vs distance $F_{z, \mathrm{SR}}(z, 0)$ spectra acquired with three different $\mathrm{CO}$ tips ( $\mathrm{CO}$ tips 1-3) above the center of a single $\mathrm{Fe}$ adatom [see Fig. S2 for corresponding energy $E_{\mathrm{SR}}(z, 0)$ curves [27] ]. While the green curve (CO tip 1) shows two pronounced minima [local physisorption force minimum $(-8 \mathrm{pN})$ and global chemisorption force minimum $(-363 \mathrm{pN})$ [23] ] separated by a repulsive barrier, CO tip 3 exhibits only a shallow but more attractive physisorption force minimum $(-26 \mathrm{pN})$ and a less attractive chemisorption force minimum $(-231 \mathrm{pN})$ without a repulsive barrier in between. In the case of CO tip 2, the chemisorption force minimum $(-448 \mathrm{pN})$ is most attractive, while the physisorption force minimum flattened out completely. The inset shows the differences of the $F_{z, \text { SR }}$ curves of CO tip 2 (3) and CO tip 1 for $100 \leq z \leq 500 \mathrm{pm}$. Note that all presented experimental curves within the main text and Supplemental Material are acquired with a step size of $\Delta z=5 \mathrm{pm}$ and plotted as connected lines for clarity. The discrete data points are plotted in Fig. S9 [27].

$F_{z, \mathrm{SR}}(z, 0)$ curves acquired with three meticulously prepared but still different $\mathrm{CO}$ tips above the center of single Fe adatoms adsorbed on $\mathrm{Cu}(111)$ (see SP2 for tip preparation and experimental details [27]). Hereby, $z$ indicates the distance to the conductance at point contact for $\mathrm{CO}$ tips on the bare $\mathrm{Cu}(111)$ surface [33] (see also SP2 [27]). As CO tip 1 approaches the single Fe adatom, it reaches a local force minimum of $F_{z, \mathrm{SR}}=-8 \mathrm{pN}$ at $z_{\text {phys }}=365 \mathrm{pm}$ (green curve in Fig. 1). It was found that this minimum is the physisorption force minimum $F_{z, \mathrm{SR}}\left(z_{\text {phys }}, 0\right)$ between the $\mathrm{CO}$ tip and the adatom, i.e., where pure physical binding and no chemical binding starts to occur [23]. Reducing the distance $z$ further results at first in slight repulsion (Pauli respulsion) and, subsequently, in a global force minimum of $F_{z, \mathrm{SR}}=-363 \mathrm{pN}$ at $z_{\text {chem }}=194 \mathrm{pm}$. The global force minimum occurs as the tip's CO molecule forms a chemical bond in this distance regime with the $\mathrm{Fe}$ adatom on the surface via hybridization [23]. Hereafter, the minimum at closer distances $z$ will be called chemisorption force minimum $F_{z, \text { SR }}\left(z_{\text {chem }}, 0\right)$ (see also SP1 [27]). Note that the CO tip remains upright during the acquisition of the $F_{z, \mathrm{SR}}(z, 0)$ spectra (see Ref. [23] and SP2 [27]).

Repeating the experiment with two other $\mathrm{CO}$ tips results in quantitatively different curves. While for $\mathrm{CO}$ tip 3 the physisorption force minimum is more attractive, it flattens out for CO tip 2. The chemisorption force minimum of $\mathrm{CO}$ tip 2 (3) is more (less) attractive compared to CO tip 1. All $\mathrm{CO}$ tips discussed here consist of a $\mathrm{CO}$ molecule bonded to a monoatomic metal tip's apex. However, the tip backgrounds, i.e., the exact shape of the metal tips' atomic structure behind the frontmost metallic atom, differ for the various metal tips (see SP2 [27]). In order to analyze the differences of the $F_{z, \mathrm{SR}}(z, 0)$ curves for the three presented CO tips, they were subtracted from each other (see inset of Fig. 1). The differential force curves do not resemble a monotonic behavior as expected if the differences in the tip backgrounds would result in a plain additive component to the $F_{z, \mathrm{SR}}(z, 0)$ curves. However, Refs. [21,48] report that the properties, i.e., the electric field of $\mathrm{CO}$ tips, strongly depend on the tip backgrounds behind the terminating $\mathrm{CO}$ molecule. Hence, we conclude that the bond between the $\mathrm{CO}$ molecule and the metal tip is different for the various $\mathrm{CO}$ tips, although all these metal tips were terminated with a single metal atom prior to $\mathrm{CO}$ functionalization, leading to quantitatively different $F_{z, \mathrm{SR}}(z, 0)$ curves (see Fig. S3 for two more CO tips [27]).

Next, Fe clusters containing 3-15 atoms are created via lateral manipulation using $\mathrm{CO}$ tips, which allows us to position each atom with atomic precision [26]. The inset of Fig. 2(a) shows the atomically resolved frequency shift $\Delta f(x, y)$ images (acquired in constant-current mode) of the investigated clusters with $\mathrm{CO}$ tip 2 . Using the constantcurrent mode allows resolving the $\mathrm{Fe}$ clusters and the $\mathrm{Cu}(111)$ substrate simultaneously with atomic resolution. The cluster's individual atoms appear as tori and adsorb on fcc positions [17]. The color of the overlaid circles indicates the reduced coordination number $c^{*}$, which is equal to the number of neighboring atoms within the Fe cluster (see SP1 [27]). The $F_{z, \mathrm{SR}}\left(z, c^{*}\right)$ curves were recorded in the most attractive $(x, y)$ positions such that the $\mathrm{CO}$ tip remains upright during the acquisition of the spectra (see SP2, SP4, and Fig. S6 [27]). All $F_{z, \mathrm{SR}}\left(z, c^{*}\right)$ spectra which were acquired on the cluster's individual atoms with various $c^{*}$ show a double-minima shape [see Fig. 2(a)] similar to the single Fe adatoms (see Fig. 1). Therefore, we suggest that the CO tip transitions from the physisorption to chemisorption regime when approaching the center of the cluster's individual atoms in the case of $c^{*}=2,3,4$, analogous to the single $\mathrm{Fe}$ adatom [23]. The central cluster atom with $c^{*}=6$ does not show the chemisorption minima that are clearly visible for $c^{*}=4,3,2,0$, but it displays a plateau at $z \approx 280 \mathrm{pm}$ and $F_{z, \mathrm{SR}} \approx 65 \mathrm{pN}$. We did not dare to approach the tip closer than $z=268 \mathrm{pm}$ to avoid damage to the tip, but we expect that the plateau would be followed by Pauli repulsion. 


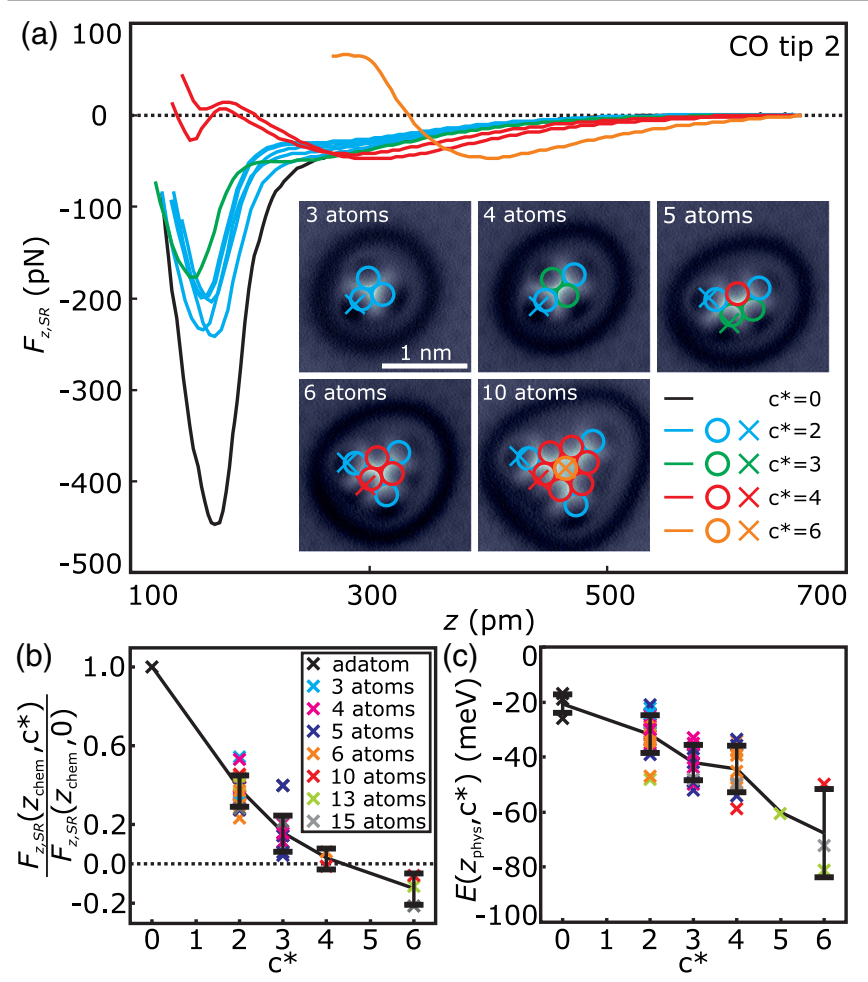

FIG. 2. (a) $F_{z, \mathrm{SR}}\left(z, c^{*}\right)$ curves above the center of a single adatom (black curve) and above the center of the individual atoms of clusters consisting of 3-6 and $10 \mathrm{Fe}$ atoms acquired with $\mathrm{CO}$ tip 2 . The atomically resolved frequency shift $\Delta f(x, y)$ images of the investigated clusters, acquired in constant-current mode, are shown in the inset. The atomic positions (circles) and the $(x, y)$ position of the $F_{z, \mathrm{SR}}\left(z, c^{*}\right)$ spectra (crosses) are overlaid (see Fig. S4 for nonoverlaid images [27]). Hereby, the circles and crosses are color coded with respect to the reduced coordination number $c^{*}$ of each atom (see SP1 [27]). (b) Chemisorption force minimum $F_{z, \mathrm{SR}}\left(z_{\mathrm{chem}}, c^{*}\right)$ normalized with respect to the chemisorption force minimum of the single adatom $F_{z, \mathrm{SR}}\left(z_{\mathrm{chem}}, 0\right)$ as a function of $c^{*}$ for $\mathrm{CO}$ tips 2-4. (c) Physisorption energy minimum $E_{\mathrm{SR}}\left(z_{\text {phys }}, c^{*}\right)$ as a function of $c^{*}$ for CO tips 2-4 [see Fig. S3 for all corresponding $F_{z, \mathrm{SR}}\left(z, c^{*}\right)$ and $E_{\mathrm{SR}}\left(z, c^{*}\right)$ curves [27] ]. The error bars in (b) and (c) indicate the standard deviation with respect to $c^{*}$.

In the following, the chemisorption force minima $F_{z, \mathrm{SR}}\left(z_{\text {chem }}, c^{*}\right)$ (i.e., the force minima at smaller $z$ value) are analyzed quantitatively as a function of $c^{*}$ (the error sources and error bars of the experimental data are discussed in SP2 [27]). The depth of the chemisorption force minima decreases clearly with reduced coordination number $c^{*}$, with $F_{z, \mathrm{SR}}\left(z_{\text {chem }}, 0\right)=-448 \mathrm{pN}, \quad F_{z, \mathrm{SR}}\left(z_{\text {chem }}, 2\right)=[-243 \mathrm{pN}$, $-195 \mathrm{pN}], F_{z, \mathrm{SR}}\left(z_{\mathrm{chem}}, 3\right)=-178 \mathrm{pN}, \quad F_{z, \mathrm{SR}}\left(z_{\mathrm{chem}}, 4\right)=$ $\{-27 \mathrm{pN}, 6 \mathrm{pN}\}$, and $F_{z, \mathrm{SR}}\left(z_{\mathrm{chem}}, 6\right)=65 \mathrm{pN}$. Repeating the experiment with two more $\mathrm{CO}$ tips results in the same observations, while the absolute values of the chemisorption force minima $F_{z, \mathrm{SR}}\left(z_{\mathrm{chem}}, c^{*}\right)$ correlate with the value of the single adatom $F_{z, \mathrm{SR}}\left(z_{\text {chem }}, 0\right)$ [see Figs. S3(a)-S3(c) [27] ]. This can be attributed to a different binding of the $\mathrm{CO}$ molecule to the various metal tip's apexes, as explained above in the case of the adatom $F_{z, \mathrm{SR}}(z, 0)$ spectra (see Fig. 1).

To enable an instructive comparison between the measurements of different $\mathrm{CO}$ tips, the $F_{z, \mathrm{SR}}\left(z, c^{*}\right)$ curves are normalized, similar to a previous atomic identification study [49], with respect to the chemisorption force minima of the single Fe adatoms $F_{z, \mathrm{SR}}\left(z_{\text {chem }}, 0\right)$ [see Figs. S3(d)S3(f) [27] ]. Figure 2(b) depicts the extracted normalized chemisorption force minima values $F_{z, \mathrm{SR}}\left(z_{\text {chem }}, c^{*}\right) /$ $F_{z, \mathrm{SR}}\left(z_{\mathrm{chem}}, 0\right)$ as a function of the reduced coordination number $c^{*}$. The plot shows that the normalized chemisorption force minimum $F_{z, \mathrm{SR}}\left(z_{\mathrm{chem}}, c^{*}\right) / F_{z, \mathrm{SR}}\left(z_{\mathrm{chem}}, 0\right)$ decreases with the reduced coordination number $c^{*}$. This is expected intuitively, as an increasing number of binding partners reduces the reactivity of the specific atom of the cluster. We interpret this observation as a direct observation of local chemical reactivity of Fe clusters. We identify the Fe cluster's corner atoms $\left(c^{*}=2\right.$ and $\left.c^{*}=3\right)$ as the active sites followed by the less reactive edge atoms $\left(c^{*}=4\right)$ with respect to their interaction with the tip's $\mathrm{CO}$ front end. Moreover, we do not find variations of the chemical reactivity of the individual atoms with a specific $c^{*}$ within the cluster for the investigated cluster sizes from 3 to 15 atoms.

Figure 2(c) shows the physisorption energy minima values $E_{\mathrm{SR}}\left(z_{\text {phys }}, c^{*}\right)$ vs reduced coordination number $c^{*}$ extracted from the corresponding $E_{\mathrm{SR}}\left(z, c^{*}\right)=-\int F_{z, \mathrm{SR}}\left(z, c^{*}\right) d z$ curves [see Figs. S3(g)-S3(i) [27] ]. Here, $z_{\text {phys }}$ indicates the position of the physisorption energy minimum (i.e., the minima at larger $z$ value). The physisorption strength $E_{\mathrm{SR}}\left(z_{\text {phys }}, c^{*}\right)$ increases with $c^{*}$. This can be explained by an increase of the attractive van der Waals interaction with increasing $c^{*}$. However, a change in the electrostatic interaction is also conceivable. The separation of these two contributions is not possible in our experiments, but could in principle be done by locally probing the electric potential of the clusters using a quantum dot sensor [50,51].

Next, the atomic contrast of Fe clusters in NCAFM is investigated. For this purpose, an atom-by-atom assembled 15 atom cluster is imaged with AFM in both constantheight and constant-current mode. The resulting atomic contrast is linked to $F_{z, \mathrm{SR}}\left(z, c^{*}\right)$ curves on specific $(x, y)$ positions on the cluster (see Fig. 3). The closest scan in constant height was performed at $z=335 \mathrm{pm}$. The corresponding deconvoluted [52,53] short-range force $F_{z, \mathrm{SR}}(x, y)$ image [see Fig. 3(b)] shows four atoms with $c^{*}=6$ in the cluster's center which are imaged as depressions (dark) with repulsion in between them. Comparing the $F_{z, \mathrm{SR}}(z, 6)$ curve on the left center atom (orange curve) with the $F_{z, \mathrm{SR}}(z)$ curve in between the atoms (magenta curve) at $z=335 \mathrm{pm}$ reveals that the atomic contrast occurs in between the physisorption minimum and the local repulsive minimum [see Fig. 3(a)]: The presence of the second repulsive force minimum results in a decrease of 


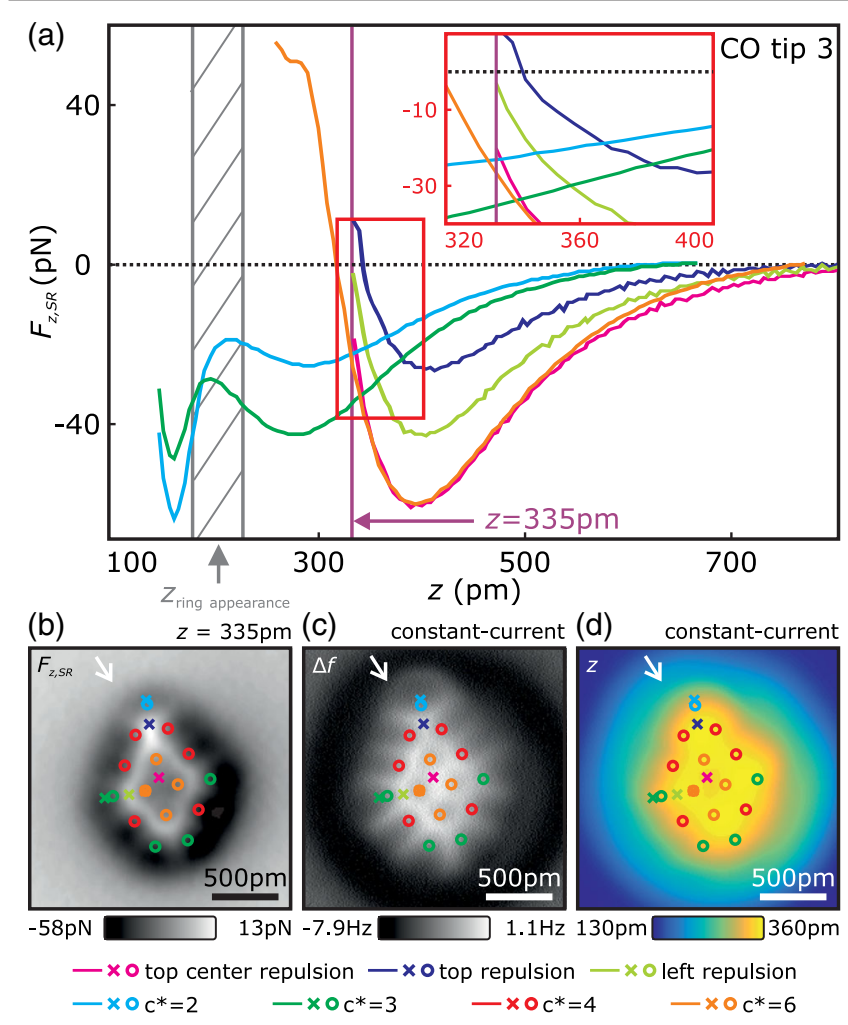

FIG. 3. (a) $F_{z, \text { SR }}(z)$ curves acquired on various positions on an atom-by-atom assembled 15 atom cluster. Enlargement of the area indicated by the red square is shown in the inset. (b) Atomically resolved vertical force image $F_{z, \mathrm{SR}}(x, y)$ at $z=335 \mathrm{pm}$ [corresponding $\Delta f_{\mathrm{SR}}(x, y)$ image and nonoverlaid images are depicted in Fig. S7 [27]]. (c),(d) Simultaneously recorded frequency shift $\Delta f(x, y)$ and topographic $z(x, y)$ images of the same spot as shown in (b) acquired in constant-current mode using $V_{\text {tip }}=-1 \mathrm{mV}$ and $\langle I\rangle=110 \mathrm{pA}$ (the same data without overlays shown in Fig. S4(j) [27]). The $(x, y)$ positions of the $F_{z, \mathrm{SR}}\left(z, c^{*}\right)$ curves (colored crosses) shown in (a), the atomic positions (colored circles), and the $(x, y)$ location of the ring appearance (white arrow) of the top atom $\left(c^{*}=2\right)$ in (c) are overlaid in panels (b)-(d). The $z$ range in which edge atoms appear as tori in (c) is indicated by $z_{\text {ring appearance }}$ in (a).

the slope of the $F_{z, \mathrm{SR}}(z, 6)$ curve (orange curve) in the distance regime of $300 \leq z \leq 360 \mathrm{pm}$ and, hence, is responsible for the appearance of the Fe atoms with $c^{*}=6$ as depressions (dark).

The cluster's corner and edge atoms $\left(2 \leq c^{*} \leq 4\right)$ also appear as depressions (dark) in constant-height mode with repulsion (bright) in between the atoms [see, e.g., the top three atoms in Fig. 3(b)]. Comparing the $F_{z, \mathrm{SR}}(z, 2)$ curve on the top corner atom (light blue curve) with the $F_{z, \mathrm{SR}}(z)$ curve acquired in the top triangle's center (dark blue curve) shows that the attractive atomic contrast occurs before the physisorption force minimum is reached [see Fig. 3(a)]. The same holds true when comparing the $F_{z, \mathrm{SR}}(z, 3)$ curve on the cluster's left corner atom (dark green curve) with the $F_{z, \text { SR }}(z)$ curve in between the atoms (light green curve).
The repulsion in between the atoms is explained by Pauli repulsion on the $\mathrm{CO}$ tip due to an increased charge density in between the atoms [see DFT-calculated charge density in Fig. S5(b) and computational details in SP3 [27] ]. Hence, the atomic contrast on the cluster's edge is a consequence of the interplay of van der Waals and electrostatic attraction on top of the atoms and Pauli repulsion in between the atoms.

In order to simultaneously achieve atomic resolution on the Fe cluster and the $\mathrm{Cu}(111)$ substrate, the constant-current mode can be used [17]. By using the constant-current mode, the tip-sample distance $z$ is reduced besides the Fe cluster to keep a certain conductance setpoint and, therefore, the $\mathrm{CO}$ tip images the $\mathrm{Cu}(111)$ surface in a distance regime where atomic resolution is achieved (see also Ref. [54]). Figures 3(c) and 3(d) show the $\Delta f(x, y)$ and the topographic $z(x, y)$ image of the same area as shown in Fig. 3(b), respectively. Comparing Fig. 3(c) with the constant-height image shown in Fig. 3(b) leads to two qualitative conclusions. First, imaging the four central atoms $\left(c^{*}=6\right)$ in constant-current mode $(330 \leq z \leq 350 \mathrm{pm})$ also results in the appearance of the atoms as depressions (dark). Second, the cluster's edge and corner atoms $\left(c^{*}=2,3,4\right)$ appear as deformed and partially open tori when imaged in constantcurrent mode, which is not the case in constant-height mode [see white arrow in Figs. 3(b) and 3(c)]. For $(x, y)$ positions above the repulsive (bright) tori of the edge and corner atoms [see, e.g., white arrow in Figs. 3(c) and 3(d)], $z$ lies within $180 \leq z_{\text {ring appearance }} \leq 230 \mathrm{pm}$ and, hence, is by 105 to $155 \mathrm{pm}$ smaller than in constant-height mode $(z=335 \mathrm{pm})$. Checking the $F_{z, \mathrm{SR}}\left(z, c^{*}\right)$ curves which were acquired on an atom of the top corner $\left(c^{*}=2\right)$ and left corner $\left(c^{*}=3\right)$ reveals that the appearance as tori in constant-current mode occurs in a distance regime [gray region in Fig. 3(a)], where the $\mathrm{CO}$ tip moves from the physisorption to the chemisorption force minimum, similar to the single adatom [23]. Hence, the tori appearance of the corner and edge atom is a result of the chemical bond formation between the CO tip and the cluster's individual atoms. In summary, the different appearances of the corner and edge atoms in constant-height and constant-current mode is a result of the absence or presence of chemical bond formation: in constant-height mode the atomic contrast is a consequence of the interplay of van der Waals, electrostatic, and Pauli interaction, while in constant-current mode chemical bonding between the tip's $\mathrm{CO}$ and the Fe atom is responsible for the atom's appearance as a torus (see Fig. S5 [27]).

Our results demonstrate that the $\mathrm{CO}$ tip allows selectively probing the chemical reactivity of individual $\mathrm{Fe}$ atoms that compose small Fe clusters. This measurement is possible as the CO tip, which is in most known cases chemically inert, transitions from the physisorption to the chemisorption regime and forms a weak chemical bond with the individual atoms when positioned above them, similar to the single $\mathrm{Fe}$ adatom. We find that the 
chemisorption strength between the CO tip and a cluster's atom increases inversely with the atom's reduced coordination number $c^{*}$ and, hence, we identify the cluster's corner and edge atoms as the cluster's active sites. Additionally, we conclude that the appearance of the Fe clusters as connected structures of tori in NCAFM images, when imaged in constant-current mode, is a result of the local chemical bond formation between the $\mathrm{CO}$ tip and the cluster's individual atoms.

The authors thank S. Matencio and F. Stilp for conducting preliminary experiments, F. Huber and A. J. Weymouth for fruitful discussions, and the Deutsche Forschungsgemeinschaft for funding within the research Project No. CRC 1277, project A02.

*julian.berwanger@ur.de

[1] H. S. Taylor, Proc. R. Soc. A 108, 105 (1925).

[2] T. Zambelli, J. Wintterlin, J. Trost, and G. Ertl, Science 273, 1688 (1996).

[3] K. Gotterbarm, F. Späth, U. Bauer, C. Bronnbauer, H.-P. Steinrück, and C. Papp, ACS Catal. 5, 2397 (2015).

[4] J. H. K. Pfisterer, Y. Liang, O. Schneider, and A. S. Bandarenka, Nature (London) 549, 74 (2017).

[5] G. Kyriakou, M. B. Boucher, A. D. Jewell, E. A. Lewis, T. J. Lawton, A. E. Baber, H. L. Tierney, M. FlytzaniStephanopoulos, and E. C. H. Sykes, Science 335, 1209 (2012).

[6] B. Yoon, H. Hakkinen, U. Landman, A. Worz, J. Antonietti, S. Abbet, K. Judai, and U. Heiz, Science 307, 403 (2005).

[7] K. S. Egorova and V. P. Ananikov, Angew. Chem., Int. Ed. 55, 12150 (2016).

[8] A. Fürstner, ACS Cent. Sci. 2, 778 (2016).

[9] C. Bolm, J. Legros, J. Le Paih, and L. Zani, Chem. Rev. 104, 6217 (2004).

[10] M. D. Morse, M. E. Geusic, J. R. Heath, and R. E. Smalley, J. Chem. Phys. 83, 2293 (1985).

[11] D. M. Cox, K. C. Reichmann, D. J. Trevor, and A. Kaldor, J. Chem. Phys. 88, 111 (1988).

[12] L.-S. Wang, X. Li, and H.-F. Zhang, Chem. Phys. 262, 53 (2000).

[13] C. P. McNary and P. B. Armentrout, Phys. Chem. Chem. Phys. 16, 26467 (2014).

[14] T. R. Albrecht, P. Grütter, D. Horne, and D. Rugar, J. Appl. Phys. 69, 668 (1991).

[15] L. Bartels, G. Meyer, and K.-H. Rieder, Appl. Phys. Lett. 71, 213 (1997).

[16] L. Gross, F. Mohn, N. Moll, P. Liljeroth, and G. Meyer, Science 325, 1110 (2009).

[17] M. Emmrich, F. Huber, F. Pielmeier, J. Welker, T. Hofmann, M. Schneiderbauer, D. Meuer, S. Polesya, S. Mankovsky, D. Ködderitzsch, H. Ebert, and F. J. Giessibl, Science 348, 308 (2015).

[18] N. Pavliček and L. Gross, Nat. Rev. Chem. 1, 0005 (2017).

[19] P. Jelínek, J. Phys. Condens. Matter 29, 343002 (2017).

[20] A. Extance, Nature (London) 555, 545 (2018).
[21] M. Ellner, N. Pavliček, P. Pou, B. Schuler, N. Moll, G. Meyer, L. Gross, and R. Peréz, Nano Lett. 16, 1974 (2016).

[22] J. Peng, J. Guo, P. Hapala, D. Cao, R. Ma, B. Cheng, L. Xu, M. Ondráček, P. Jelínek, E. Wang, and Y. Jiang, Nat. Commun. 9, 122 (2018).

[23] F. Huber, J. Berwanger, S. Polesya, S. Mankovsky, H. Ebert, and F. J. Giessibl, Science 366, 235 (2019).

[24] N. Moll, L. Gross, F. Mohn, A. Curioni, and G. Meyer, New J. Phys. 12, 125020 (2010).

[25] N. Moll, L. Gross, F. Mohn, A. Curioni, and G. Meyer, New J. Phys. 14, 083023 (2012).

[26] J. Berwanger, F. Huber, F. Stilp, and F. J. Giessibl, Phys. Rev. B 98, 195409 (2018).

[27] See Supplemental Material at http://link.aps.org/supplemental/ 10.1103/PhysRevLett.124.096001 for the used terminology, the experimental details, the computational details, a discussion of the adsorption geometry of $\mathrm{CO}$ molecules on $\mathrm{Fe}$ clusters, further $F_{z, \mathrm{SR}}\left(z, c^{*}\right)$ curves with two more CO tips corresponding to Fig. 1 including the corresponding $E_{\mathrm{SR}}\left(z, c^{*}\right)$ curves, all $F_{z, \mathrm{SR}}\left(z, c^{*}\right)$ curves for $\mathrm{CO}$ tips 2-4 corresponding to Fig. 2 including all atomically resolved AFM images of the studied Fe clusters, the contrast formation analysis for the 10 atom $\mathrm{Fe}$ cluster, the corresponding vertical $F_{z \text {,DFT }}$ and lateral force $F_{x, \mathrm{DFT}}$ curves which are used to exclude an influence of $\mathrm{CO}$ bending, and the nonoverlaid image of Fig. 3(b) including the corresponding $\Delta f$ image, which includes Refs. [28-47].

[28] F. J. Giessibl, Appl. Phys. Lett. 73, 3956 (1998).

[29] J. Welker and F. J. Giessibl, Science 336, 444 (2012).

[30] A. Biedermann, W. Rupp, M. Schmid, and P. Varga, Phys. Rev. B 73, 165418 (2006).

[31] N. N. Negulyaev, V. S. Stepanyuk, L. Niebergall, P. Bruno, W. Auwärter, Y. Pennec, G. Jahnz, and J. V. Barth, Phys. Rev. B 79, 195411 (2009).

[32] F. J. Giessibl, H. Bielefeldt, S. Hembacher, and J. Mannhart, Appl. Surf. Sci. 140, 352 (1999).

[33] M. Schneiderbauer, M. Emmrich, A. J. Weymouth, and F. J. Giessibl, Phys. Rev. Lett. 112, 166102 (2014).

[34] Z. Majzik, M. Setvín, A. Bettac, A. Feltz, V. Cháb, and P. Jelínek, Beilstein J. Nanotechnol. 3, 249 (2012).

[35] L. Nony, F. Bocquet, F. Para, and C. Loppacher, Phys. Rev. B 94, 115421 (2016).

[36] F. J. Giessibl, Phys. Rev. B 56, 16010 (1997).

[37] F. J. Giessibl, Rev. Sci. Instrum. 90, 011101 (2019).

[38] W. L. Bragg, Lond. Edinburgh Dublin Philos. Mag. J. Sci. 28, 355 (1914).

[39] F. J. Giessibl, Rev. Mod. Phys. 75, 949 (2003).

[40] S. Morita, F. J. Giessibl, and R. Wiesendanger, Noncontact Atomic Force Microscopy, 2nd ed. (Springer-Verlag, Berlin, 2009).

[41] G. Kresse and J. Hafner, Phys. Rev. B 47, 558 (1993).

[42] G. Kresse and J. Hafner, J. Phys. Condens. Matter 6, 8245 (1994).

[43] J. P. Perdew, K. Burke, and M. Ernzerhof, Phys. Rev. Lett. 77, 3865 (1996).

[44] S. Grimme, J. Antony, S. Ehrlich, and H. Krieg, J. Chem. Phys. 132, 154104 (2010).

[45] R. V. G. Ewens and M. W. Lister, Trans. Faraday Soc. 35, 681 (1939). 
[46] J. T. Lyon, P. Gruene, A. Fielicke, G. Meijer, and D. M. Rayner, J. Chem. Phys. 131, 184706 (2009).

[47] G. L. Gutsev, C. W. Bauschlicher, and L. Andrews, J. Chem. Phys. 119, 3681 (2003).

[48] F. Schulz, J. Ritala, O. Krejčí, A. P. Seitsonen, A. S. Foster, and P. Liljeroth, ACS Nano 12, 5274 (2018).

[49] Y. Sugimoto, P. Pou, M. Abe, P. Jelinek, R. Pérez, S. Morita, and Ó. Custance, Nature (London) 446, 64 (2007).

[50] C. Wagner, M. F. B. Green, P. Leinen, T. Deilmann, P. Krüger, M. Rohlfing, R. Temirov, and F. S. Tautz, Phys. Rev. Lett. 115, 026101 (2015).
[51] C. Wagner, M. F. B. Green, M. Maiworm, P. Leinen, T. Esat, N. Ferri, N. Friedrich, R. Findeisen, A. Tkatchenko, R. Temirov, and F. S. Tautz, Nat. Mater. 18, 853 (2019).

[52] J. E. Sader and S. P. Jarvis, Appl. Phys. Lett. 84, 1801 (2004).

[53] J. E. Sader, B. D. Hughes, F. Huber, and F. J. Giessibl, Nat. Nanotechnol. 13, 1088 (2018).

[54] D. Martin-Jimenez, S. Ahles, D. Mollenhauer, H. A. Wegner, A. Schirmeisen, and D. Ebeling, Phys. Rev. Lett. 122, 196101 (2019). 